\title{
Revealing the Role of Epithelial Mechanics and Macrophage Clearance during Pulmonary Epithelial Injury Recovery in the Presence of Carbon Nanotubes
}

\author{
Dedy Septiadi, * Wildan Abdussalam, Laura Rodriguez-Lorenzo, Miguel Spuch-Calvar, \\ Joël Bourquin, Alke Petri-Fink, and Barbara Rothen-Rutishauser*
}

Wound healing assays are extensively used to study tissue repair mechanisms; they are typically performed by means of physical (i.e., mechanical, electrical, or optical) detachment of the cells in order to create an open space in which live cells can lodge. Herein, an advanced system based on extensive photobleaching-induced apoptosis; providing a powerful tool to understand the repair response of lung epithelial tissue, consisting of a small injury area where apoptotic cells are still intact, is developed. Notably, the importance of epithelial mechanics and the presence of macrophages during the repair can be understood. The findings reveal that individual epithelial cells are able to clear the apoptotic cells by applying a pushing force, whilst macrophages actively phagocytose the dead cells to create an empty space. It is further shown that this repair mechanism is hampered when carbon nanotubes (CNTs) are introduced: formation of aberrant (i.e., thickening) F-actins, maturation of focal adhesion, and increase in traction force leading to retardation in cell migration are observed. The results provide a mechanistic view of how CNTs can interfere with lung repair.

Tissue repair following injury involves cell migration, cell spreading, and cell division ${ }^{[1]}$ in which cell mechanics has been shown playing a very important role. ${ }^{[2,3]}$ Alteration of mechanical properties of the cells can lead to abnormal injury recovery responses and it has been speculated to be responsible in the progression of mechano-associated diseases such as idiopathic pulmonary fibrosis. ${ }^{[4]}$ In vitro wound healing assays based on

Dr. D. Septiadi, Dr. L. Rodriguez-Lorenzo, Dr. M. Spuch-Calvar,

J. Bourquin, Prof. A. Petri-Fink, Prof. B. Rothen-Rutishauser

Adolphe Merkle Institute

University of Fribourg

Chemin des Verdiers 4, 1700 Fribourg, Switzerland

E-mail: dedy.septiadi@unifr.ch; barbara.rothen@unifr.ch

Dr. W. Abdussalam

Department of High Energy Density

Helmholtz-Zentrum Dresden-Rossendorf

Bautzner Landstraße 400, 01328 Dresden, Germany

Prof. A. Petri-Fink

Department of Chemistry

University of Fribourg

Chemin du Musée 9, 1700 Fribourg, Switzerland removal of large tissue area (i.e., to create open space), either by cell scratching ${ }^{[5]}$ or by means of photoablation, ${ }^{[3,6]}$ have been widely used to study tissue repair mechanisms. However, they are unsuitable for studying the repair response of the tissue consisting of a small injury site where dead cells, due to apoptotic or necrosis, are still intact or present (i.e., no open space is available). In this paper, we report an advanced wound healing system based on extensive photobleaching-induced apoptosis which is able to mimic closely pulmonary epithelial (micro) injury and examine the wound healing response in the presence of macrophages (Figure 1a). We were able to understand the importance of (epithelial) protrusion mechanics (i.e., pushing force) and phagocytic clearance of apoptotic cells by macrophages during the tissue repair in an advanced 3D lung cell model. Moreover, we also successfully demonstrated that epithelial wound healing was hampered when high aspect ratio nanomaterials such as carbon nanotubes (CNTs) were introduced into the system.

We used the extensive photobleaching module available on the Zeiss LSM 710 confocal microscope system to produce epithelial injuries (i.e., by applying similar technique used in fluorescence recovery after photobleaching, but performed with longer exposure time, Figure S1, Supporting Information). We have chosen the A549 alveolar epithelial cell line since they can undergo tissue repair in wound-healing assays ${ }^{[7]}$ and have been reported to contribute to CNT-induced fibrogenesis. ${ }^{[8]}$ The selected wound area corresponds to five different fields of exposures (see the Experimental Section) was generated in epithelial monolayers by exciting the cell region continuously with a $405 \mathrm{~nm}$ ultraviolet (UV) continuous wave laser. The laser is focused through a $20 \times$ objective lens with typical parameters of: $1.94 \mathrm{~s}$ scanning time, 1000 iteration, $30 \mathrm{~mW}$ laser power, and numerical aperture of the objective 0.8 . For example, an injury site with the size of $83 \mu \mathrm{m} \times 83 \mu \mathrm{m}$ in a $425.10 \mu \mathrm{m} \times 425.10 \mu \mathrm{m}$ field of view corresponds to around 30 to 40 epithelial cells which are damaged (Figures S2 and S3, Supporting Information). To investigate possible membrane damage after intense laser irradiation (i.e., cell death indicator), we introduced DAPI, a typically membrane impermeable dye that is commonly used 
a

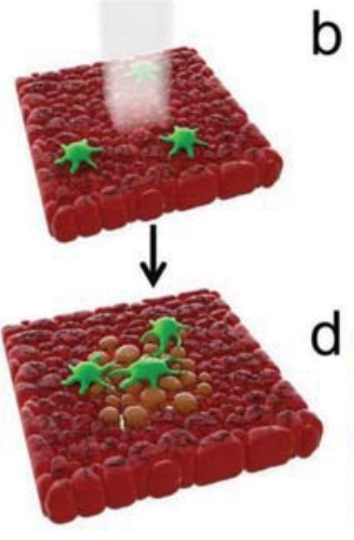

e

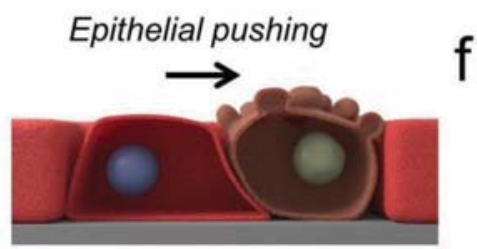

g

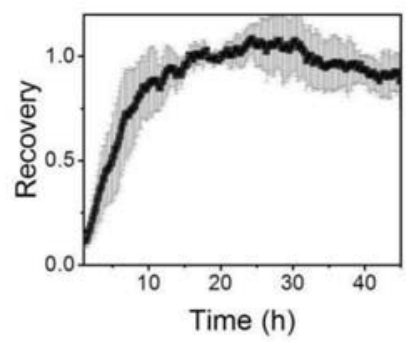

Oh

f
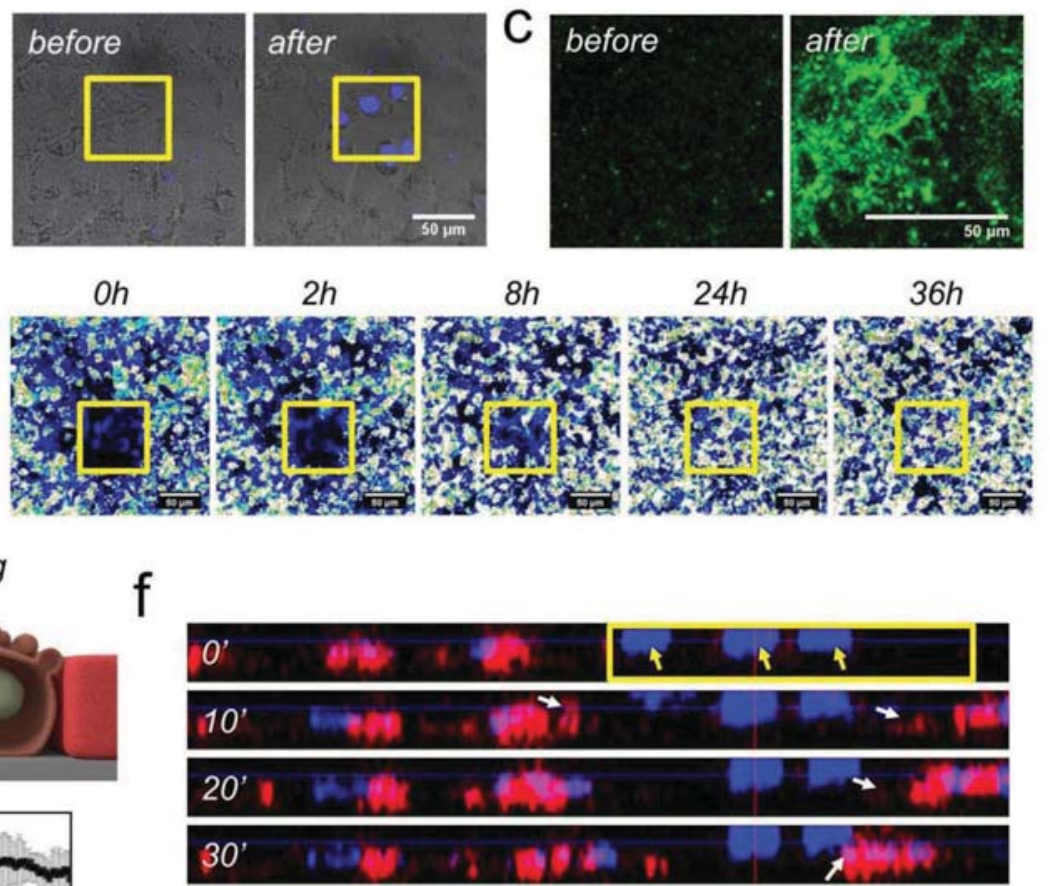

\section{0}

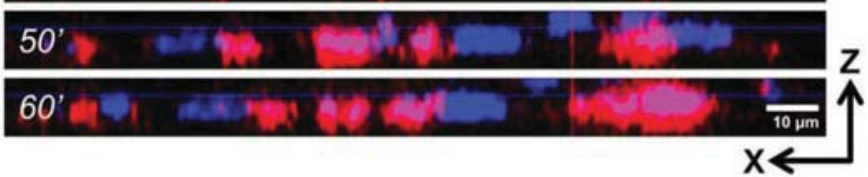

Figure 1. a) Schematic representation of epithelial injury recovery in the presence of CNTs and macrophages. Generation of epithelial injury was performed by means of an extensive photobleaching module. b) Nonpermeable membrane dye (i.e., DAPI, blue) and c) Annexin V staining (green) was used to investigate the nature of cell death upon the extensive UV photobleaching. The yellow square indicates the injury site. d) Time-lapse maximum intensity projection images of epithelial cells showing the recovery of injury over time through invasion of living cells into wound area. Images were represented as intensity-coded pictures. e) Schematic showing removal of dead cells through epithelial mechanics (i.e., pushing). f) Side view timelapse images showing the pushing of apoptotic cells (yellow square and arrows) by live epithelial cells. We show that protrusion mechanics (white arrows) plays an important role for clearance of the dead cells. Blue and red show the cell nuclei and cytoplasm, respectively. g) Rate of injury recovery corresponds to a wound size $83 \mu \mathrm{m} \times 83 \mu \mathrm{m}$ calculated by means of image processing. The black line shows the average value whilst grey line denotes the standard deviation.

for staining the nuclei of fixed cells, and collected its corresponding fluorescence signal. Figure $1 \mathrm{~b}$ shows a comparison of the epithelial monolayer and injury site (yellow square) before and after the extensive photobleaching. We observed that after UV irradiation, a clear and distinctive DAPI signal appears in the cell nuclei of the irradiated area (blue) accompanied by cell shrinkage as well as the formation of apoptotic membrane blebbing and loss of cell-cell adhesion, indicating cell death through apoptosis (Figure S4, Supporting Information). To confirm this finding, Annexin V staining was performed. We detected the presence of highly fluorescent Annexin V conjugate on the outer parts of cell membranes, which serves as an indication of cell apoptosis (Figure 1c). It is worthwhile to emphasize that these dead cells still maintain their adhesion property. No detachment of dead cells was observed even after prolongation of culture time; therefore, no open space was created, which is a very important base for the development of a novel model for wound healing (Figure S5, Supporting Information).
Our very first objective of this study was to understand whether live epithelial cells could migrate to an area occupied by dead cells. We hypothesize that these dead cells behave like a scar that must be removed and cleared before healthy cells can occupy the space created (Figure 1e; Figure S5, Supporting Information). To ease visualization of the epithelial monolayers, we fluorescently labeled the nuclei and cell cytoplasm using commercially available membrane-permeable fluorophores, Hoechst 33342, and Vybrant DiD cell-labeling solution, respectively. To study the injury recovery response of epithelial monolayers after the injury experiment, we monitored the cells under fluorescence confocal microscope using z-stack and time-lapse imaging.

Figure 1d shows selected time frames of mean fluorescence intensity images of the epithelial monolayers. Immediately following laser irradiation, we observed the presence of adherent dead cells that possess a moderate fluorescence signal (yellow square) due to partial fluorescence photobleaching. Furthermore, we noticed that the first migration of live cells to the 
wound area took place between $30 \mathrm{~min}$ to $1 \mathrm{~h}$ after the wound was formed (see Video S1, Supporting Information). Cellular invasion was observed from all directions and individual cell migration mostly dominated the recovery process, beginning with expansion of the cell membrane (i.e., filopodia formation) of epithelial cells and retraction of the cell body. Inside the injury site, this protrusion produced a substantial pushing force on the static apoptotic cells, leading to a faster loss of their adhesion (Figure 1e,f). As a consequence, when the dead cells were detached, a new space is automatically available for the cells to occupy. Using image processing-based recovery quantification (Figure S3, Supporting Information) we were able to show that removal of $\approx 30$ to 40 apoptotic cells from a wound area (size $83 \mu \mathrm{m}$ x $83 \mu \mathrm{m}$ ) and full recovery of monolayer which follows a sigmoidal (exponential) curve was achieved after $20 \mathrm{~h}$ (see Figure 1g; Video S1, Supporting Information). Furthermore, we found out that the speed of recovery is also dependent on the injury size; the larger the size, the slower the recovery (Figure S6, Supporting Information).

We hypothesize that the driving force of this invasion (i.e., directed individual migration to the injury area) is due to two reasons: (i) production of chemokines by dead epithelial cells which subsequently serve as chemoattractants for live cells to migrate or (ii) the loss of contact inhibition, ${ }^{[9]}$ especially between dead cells inside the injury site and live cells in the proximity. Interleukin 8 (IL8) also known as chemokine (C-X-C motif) ligand 8 (CXCL8) is one of the key chemokines produced by epithelial cells and macrophages and it has been shown to act as the primary chemoattractant during inflammation and tissue repair. ${ }^{[10]}$ To investigate the production or release of IL8 in cell culture media after injury, we collected the culture supernatant and performed enzyme-linked immunosorbent assay (ELISA). Our data show that there is indeed a slight increase of IL8 production $24 \mathrm{~h}$ after injury in comparison to control cells (i.e., cells without injury; Figure 2a). Lower values of IL8 production can be attributed to release of IL8 from the small population of cells present in the induced injury (i.e., $0.48 \mathrm{~cm}^{2}$ ) which is only a fraction of the total size of the epithelial layer cultured in a four-well plate (surface area of $1.7 \mathrm{~cm}^{2}$ ).

The role of individual epithelial cell mechanics in the clearance and reoccupation of the site was modelled by applying a Monte Carlo simulation on the migration model developed by Drasdo et al. and Fong et al (see Section S1 of the Supporting Information for details). ${ }^{[11,12]}$ Figure $2 \mathrm{~b}$ and Video S2 (Supporting Information) shows the realization of simulation results for individual epithelial cell migration toward the
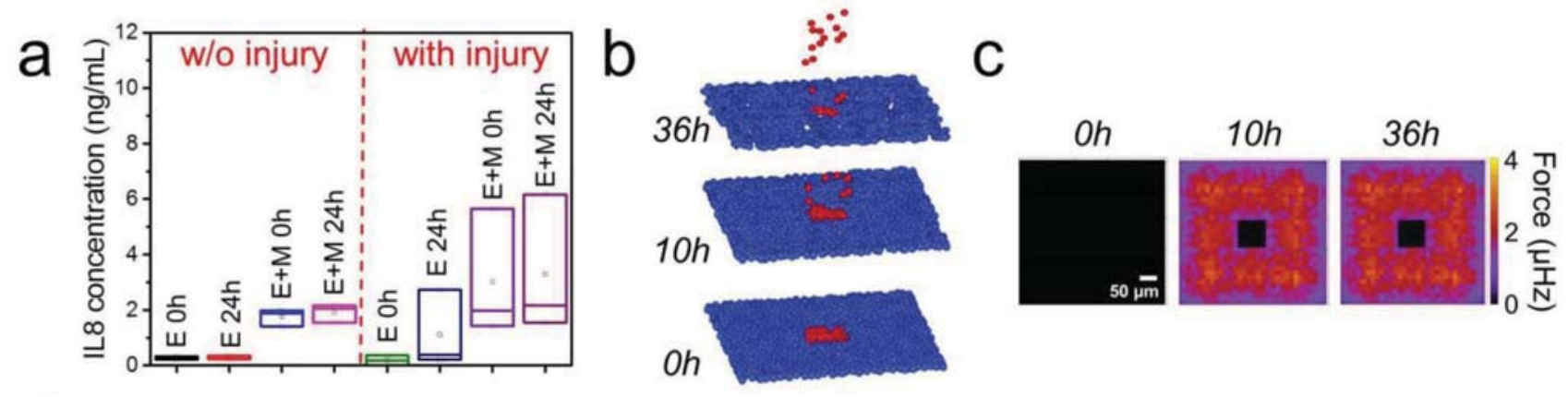

d

Oh

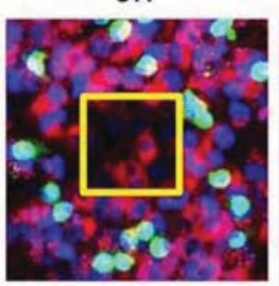

$2 h$
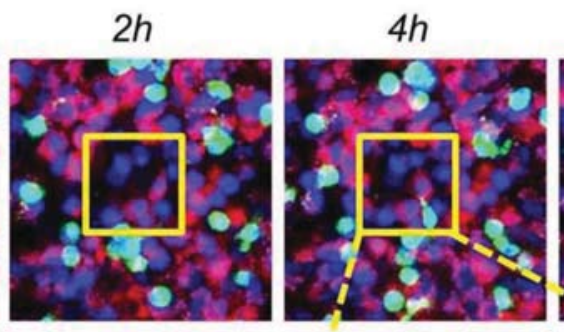

$8 h$

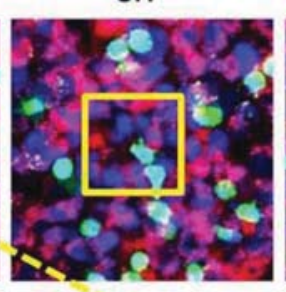

$24 h$

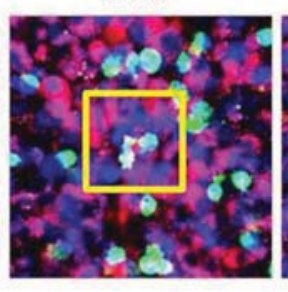

$36 h$

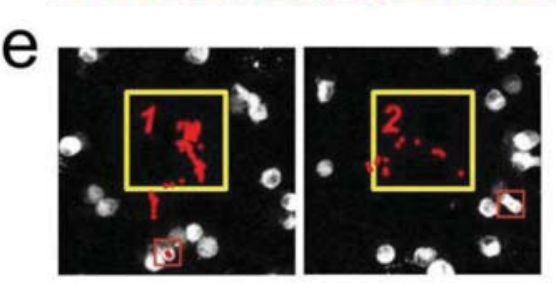

$f$
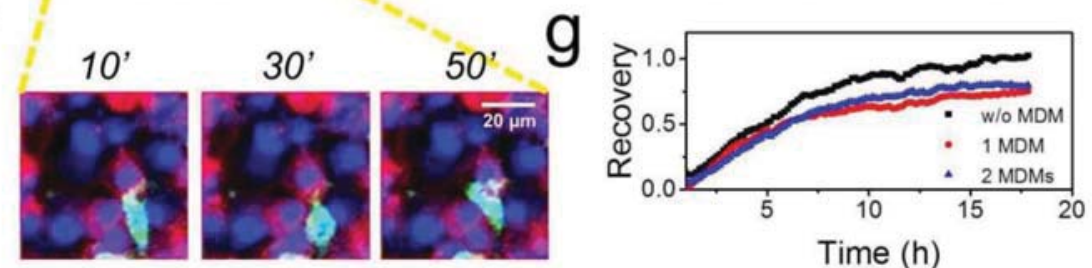

Figure 2. a) Production of Interleukin 8 (IL8) chemokine in the presence and in the absence of injury at two different time points (i.e., 0 and 24 h) in the epithelial and macrophage coculture systems measured by ELISA. E and M denotes epithelial and macrophages, respectively. b) Computer simulation displaying the role of epithelial mechanics in the recovery of injury: live epithelial cells (blue) push apoptotic cells (red) allowing detachment of dead cells from the substrate and subsequent filling of the space. c) Computed force exerted by live epithelial cells to their neighbors. d) Time-lapse images showing the involvement of macrophages (MDMs; green) in the clearance of apoptotic cells. The cell nuclei and cytoplasm are shown in blue and red, respectively. The yellow square denotes the injury area. e) Cell tracking showing the trajectories (in red) of two different MDMs migrating toward injury sites. f) Clearance of apoptotic cells through phagocytosis. g) Graph depicts different rate of recovery in the absence/presence of MDMs. 
wound area. Briefly, we divided the cells region into three areas: (i) innermost, the living cells close to the wound; (ii) outermost, the ones close to the edge of substrate; (iii) intermediate, the cells in between these two regions. At the initial condition, $t=0 \mathrm{~h}$ an ensemble of cells were randomly arranged on a 2D area such that the interdistance between cells was greater than the cell radius $\left(r_{\min }>R\right)$ (see Figure $2 \mathrm{~b}$ ). Due to the presence of pressure exerted by cells on the edge of the substrate to their adjacent cells, the latter subsequently migrate toward the center of the cell ensemble. As a result, the apoptotic cells present at the interface adjacent to the innermost cell region are pushed out (see Figure 2b, $t=10 \mathrm{~h}$ ) when the interdistance minimum is less than the distance of the innermost living cells and the outermost apoptotic cells $\left(r_{\min }<r_{\text {ld }}\right)$. Since living cells in the innermost region move faster than cells in the intermediate cell region, they eventually push all the apoptotic cells out of the ensemble (Figure $2 \mathrm{~b}$ at $t=36 \mathrm{~h}$ ).

The migration rates of living cells can be understood from the distribution of forces on the cells (see force maps in Figure 2c). At $t=0 \mathrm{~h}$, all cells are at rest and thus there are no forces around the cells. As the living cells begin to move, given that the intermediate cells consider forces due to their next-nearest neighbors (Figure $2 \mathrm{c}, t=10 \mathrm{~h}$ ), the exerted forces of intermediate cells are stronger than the contributions from the other cell populations. Consequently, the migration probability of an individual cell $\left(\exp \left(-\left[F\left(\bar{S}_{i}\right)-F\left(S_{i}\right)\right]\right)\right)$; see details in the Supporting Information) which occupies either the innermost or outermost regions is higher than the cell which occupies the intermediate one. This allows the innermost cell population to rapidly push the outer apoptotic cells out (Video S3, Supporting Information). The migration occurs until full removal of the apoptotic cells in which its distribution of forces is shown on Figure 2c for $t=36 \mathrm{~h}$.

In human and animal biological systems such as respiratory organs, it is well-known that the clearance of foreign toxic compounds and apoptotic dead cells is performed mainly by macrophages. Our data have demonstrated that in a context of epithelial injury recovery, epithelial cells were indeed able to remove/detach the dead cell by applying pushing forces through protrusion mechanics. Herein, we showed that the presence of macrophages could influence the healing speed because of their phagocytic nature and therefore clearing of apoptotic cells. We constructed coculture systems containing epithelial cells and human blood monocyte-derived macrophages (MDMs) and performed a similar wound healing experiment as described earlier. For better visualization and distinction between the two types of cells, we stained macrophages with Vybrant DiI (green) and epithelial cells with Vybrant DiD cell-labeling solution as well as Hoechst 33342 (red and blue). Using time-lapse microscopy, we observed simultaneous directed migration of small numbers of MDMs and invasion of neighbor epithelial cells to the injury area starting $\approx 1 \mathrm{~h}$ after wound formation (Figure 2d; Video S4, Supporting Information). We attributed this migration to the higher production of IL8 after injury generation especially in the presence of macrophages (Figure 2a). Using image tracking analysis, we found that the macrophages which reside in close proximity to the wound area traveled to the injury site at an approximate speed of $3.68 \mu \mathrm{m} \mathrm{min}{ }^{-1}$ (Figure 2e). It is almost four-times faster than the movement of "nonattracted" macrophages $\left(0.92 \mu \mathrm{m} \mathrm{min}^{-1}\right)$ or the movement of cells in the absence of stimuli reported in the literature (i.e., $\left.<1.00 \mu \mathrm{m} \mathrm{min}{ }^{-1}\right) \cdot{ }^{[13]}$ Our reported value is almost half of the fastest migration speed that has ever been measured in vivo, which is over $10 \mu \mathrm{m} \mathrm{min}{ }^{-1}$ when macrophages are attracted to an induced wound in a fish model. ${ }^{[14]}$ The first phagocytic event in the injury site was captured around $4 \mathrm{~h}$ after beginning the experiment (Figure 2f) and continued over the next $40 \mathrm{~h}$. We also noticed that macrophages were able to pull out apoptotic cells from the injury site and subsequently performed phagocytosis of the debris outside the site (Figure S7, Supporting Information). Analysis of the recovery rate as function of number MDMs involved in the clearance showed a slight enhancement of epithelial recovery particularly when more MDMs (two vs one) were present (Figure $2 \mathrm{~g}$ ), however, the reported value was still less than the recovery value of the control sample when no MDMs were involved. We attribute this to a possible competitive effect that exists between live epithelial and macrophages, as both cells need to occupy the injury site in order to clear the dead cells. In addition, we noticed that the number of MDMs reduced over time as the cells underwent apoptosis and subsequently detached from the epithelial monolayer after $36 \mathrm{~h}$. Nevertheless, our result provides an important finding highlighting the role of macrophages in helping the clearance of apoptotic cells and providing empty space for the epithelial cells.

Repeated inhalation of cigarette smoke ${ }^{[15]}$ or occupational pollutants such as metals have been associated with idiopathic pulmonary fibrosis. ${ }^{[16]}$ Recently the exposure to high aspect ratio nanomaterials like multiwalled carbon nanotubes (CNTs) have been shown to be able to induce cell killing (mainly through apoptosis) and further form microinjuries in epithelial lung tissue, leading to the development of pulmonary fibrosis in animal models. ${ }^{[17,18]}$ Once the tissue is injured, the regeneration (lung repair) will start to take place; however, this procedure generally takes place over a long time (i.e., a couple of weeks). ${ }^{[19]}$ In our experiment, we simulated the repair of epithelial injury in the presence of CNTs. We chose hydrophobic Mitsui-7 CNTs that were stabilized in a bovine serum albumin (BSA)-containing dispersion as our testing material. Electron images of the CNT dispersion as depicted in Figure S8 of the Supporting Information show the average length and width $\approx 5.6 \mu \mathrm{m}$ and $60 \mathrm{~nm}$, respectively. We simultaneously introduced these BSA-stabilized CNTs at two initial concentrations: 5 and $10 \mu \mathrm{g} \mathrm{mL} \mathrm{m}^{-1}$ (i.e., below subtoxic concentration ${ }^{[20]}$ ) to our epithelial monolayers (i.e., without MDMs), generated the wound and monitored the response of the cells over $40 \mathrm{~h}$ after the injury formation. A direct comparison with negative control systems (i.e., no CNTs) was also performed.

Figure 3a shows different kinetics of injury recovery in the absence and in the presence of CNTs (see also Video S5 of the Supporting Information for visualization). We found that CNTtreated epithelial cells possessed slow recovery (for both halftime and full-time recovery) in comparison to untreated cells and as expected, this process is also concentration dependent (Figure 3a). We hypothesized that this slow recovery is related to a slight retardation of individual cell migration, as has been shown occurring in CNT-treated cells as reported by Fanarraga and co-workers. ${ }^{[21]}$ To confirm this finding, we investigated any 


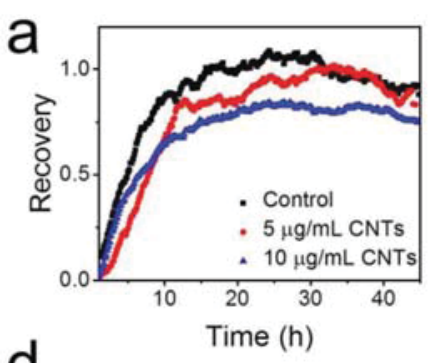

d

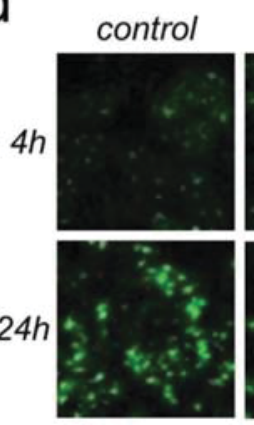

$5 \mu \mathrm{g} / \mathrm{mL}$

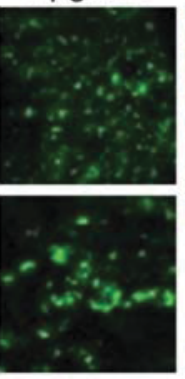

b
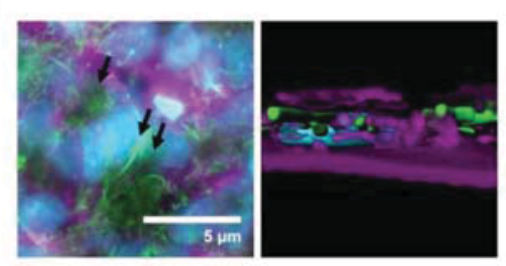

e
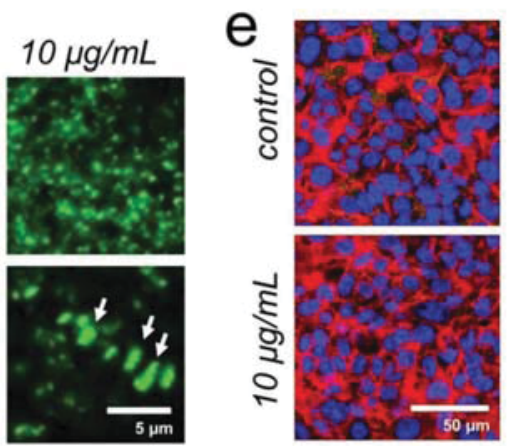

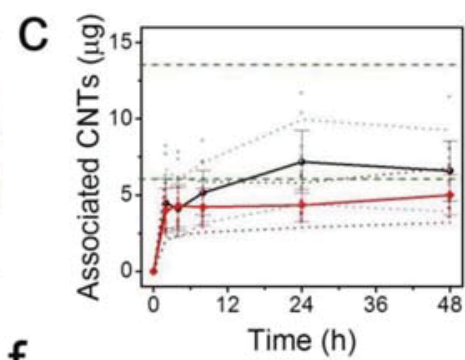

f

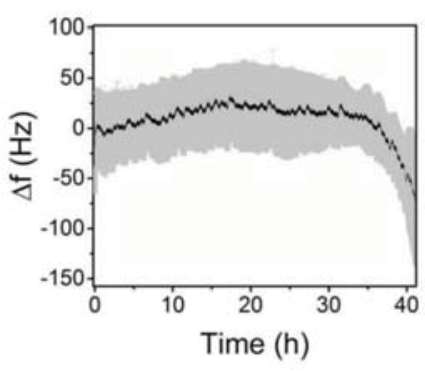

Figure 3. a) Graph depicting different recovery kinetics in the absence and in the presence of CNTs. Slow recovery was found on CNT-treated cells, which can be associated with minor retardation of cell migration. b) Enhanced dark-field fluorescence image of associated CNTs on epithelial cells and the corresponding 3D rendered image. CNTs are shown in green, whilst nuclei and F-actins cytoskeleton appear in cyan and magenta, respectively. Initial concentration (i.e., administered dose) of CNTs is $10 \mu \mathrm{g} \mathrm{mL} \mathrm{L}^{-1}$. c) In vitro concentration of associated CNTs on epithelial cells was measured by means of UV-vis-NIR spectroscopy. Increase of associated CNTs over time was observed. The red and black lines show two different administered doses, 5 and $10 \mu \mathrm{g} \mathrm{mL}^{-1}$. The green dashed lines represent the initial mass of CNTs, 7.5 and $15 \mu \mathrm{g}$, for 5 and $10 \mu \mathrm{g} \mathrm{mL}^{-1}$, respectively. d,e) Fluorescence images of paxillin focal adhesions (d) and F-actin cytoskeletons (red) (e). Maturation of paxillin focal adhesions (white arrows) and formation of aberrant actin cytoskeleton due to cellular association of CNTs was observed. f) Adhesion dynamics of CNT-treated epithelial cells visualized by quartz crystal microbalance. The black and gray lines represent the mean and standard deviation, respectively.

possible effect of CNTs on cytoskeletal architecture (e.g., F-actin assembly), adhesion properties (i.e., formation and maturation of focal adhesion) and cellular traction force. Protrusion of the F-actin cytoskeleton, cell adhesion, and generation of traction force are the three major components which are responsible for cell locomotion. ${ }^{[22]}$

Using enhanced darkfield and fluorescence imaging, we were able to locate CNTs on cell bodies. Our results confirm that CNTs were either strongly attached to the cell membrane or partially internalized (Figure 3b; Figure S9, Supporting Information). By means of optical spectroscopy ${ }^{[23]}$ (Figure S10, Supporting Information), the amount of these cell-associated CNTs (i.e., attached and internalized) were successfully quantified, and the intracellular CNT concentration was found to increase over the time due to a longer incubation time (Figure 3c). We believe that the high degree of associated CNTs (i.e., more than $60 \%$ of administered CNTs interacting with cells) have a tendency to entangle with cytoskeleton components, and in our case with F-actins (Figure $3 \mathrm{~b}$ ). Our fluorescence microscopy data as seen in Figure 3d and Figure S11 (Supporting Information) confirms the formation of aberrant F-actins (i.e., thickening) and is an indication of cytoskeletal damage. ${ }^{[24]}$ Disruption of F-actin microfilaments leading to retardation of cellular migration has already been reported in two cell lines after incubation with different types of nanoparticles. ${ }^{[25,26]}$ In addition, the previous example has shown that the interaction of CNTs with microtubules produces centrosomal mispositioning, disrupting the nuclear-centrosomal axis and hence hindering cancer cell migration. ${ }^{[21]}$
To further investigate the effect of CNTs on cell adhesion, we performed microscopy experiments of fluorescently-labeled paxillin focal adhesion of cells cultured with and without CNTs (see result in Figure 3e). Our image analysis showed an increase in the size of paxillin dense regions in CNT-treated cells in comparison to control ones (Figure S12, Supporting Information), confirming maturation of paxillin focal adhesions which could lead to stronger cell adhesion. We also performed quartz crystal microbalance experiments (Figure S13, Supporting Information) as suggested by Gryte et al. and Wang et al. ${ }^{[27,28]}$ We did not identify any significant change in the adhesion property of the entire monolayer, both in the absence and in the presence of CNTs, except after $36 \mathrm{~h}$, which can be attributed to cell death due to long incubation (Figure $3 \mathrm{f}$ ). By contrast, the contribution of average force on the substrate exerted by each individual was measured by micropillar-based traction force microscopy ${ }^{[29,30]}$ (Figure 4a,d). By measuring the deflection of individual pillars, the average force exerted by the cells on the pillars can be determined. Our results suggest that CNT-treated individual cells possess almost twofold higher traction force in comparison to nontreated ones (Figure 4e). During simulation, we attributed the presence of CNTs in our systems as a dissipation/disturbance factor (see details in the Supporting Information). As the forces exerted and experienced by cells increases (as also shown from traction force data), the acceptance probability decreases and as a result slows down the cell migration (Videos S6-S9, Supporting Information), preventing fast injury recovery. All of these findings provide evidence for retardation of individual cell migration through the disruption of cytoskeletal components, 

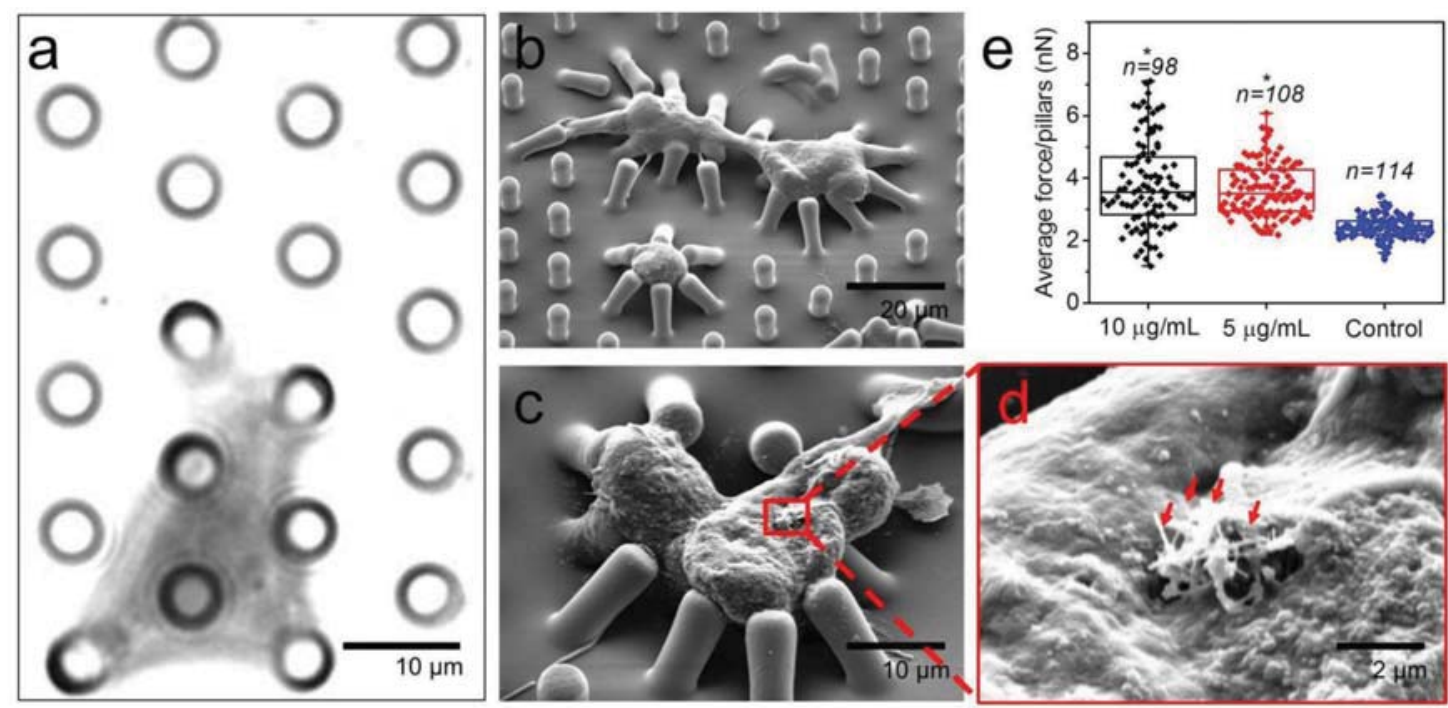

Figure 4. Micropillar assay-based traction force microscopy. a) Bright-field and b) scanning electron image of nontreated epithelial cells grown on micropillars showing the bend of the pillars due to force exerted by a single cells. c,d) Scanning electron image of CNT-treated cells and the enlargement area showing the associated CNTs in the cells (red arrow). e) Average cell traction force/pillars for different conditions. $n$ denotes number of individual cells. One-way ANOVA, $* p<0.05$.

changes in adhesion through maturation of focal adhesions and an increase in traction force (which is due to the presence of CNTs).

Finally, we hypothesized that presence of CNTs would become a distraction for macrophages to perform their function; possible competition between clearing the apoptotic cells or foreign materials (i.e., CNTs) would occur. At the same time, the number of CNTs internalized by epithelial cells would be reduced due to the phagocytic nature of macrophages which preferentially perform the uptake of CNTs, restoring wound healing by epithelial cells. To prove this assumption in our coculture systems (i.e., epithelial and macrophages), we added CNT suspension and subsequently performed the wound healing experiment. Using time-lapse bright-field imaging, the phagocytic activity of macrophages involved in clearance of CNTs was revealed. Figure 5a-c shows selected frames (i.e., 0, 4,
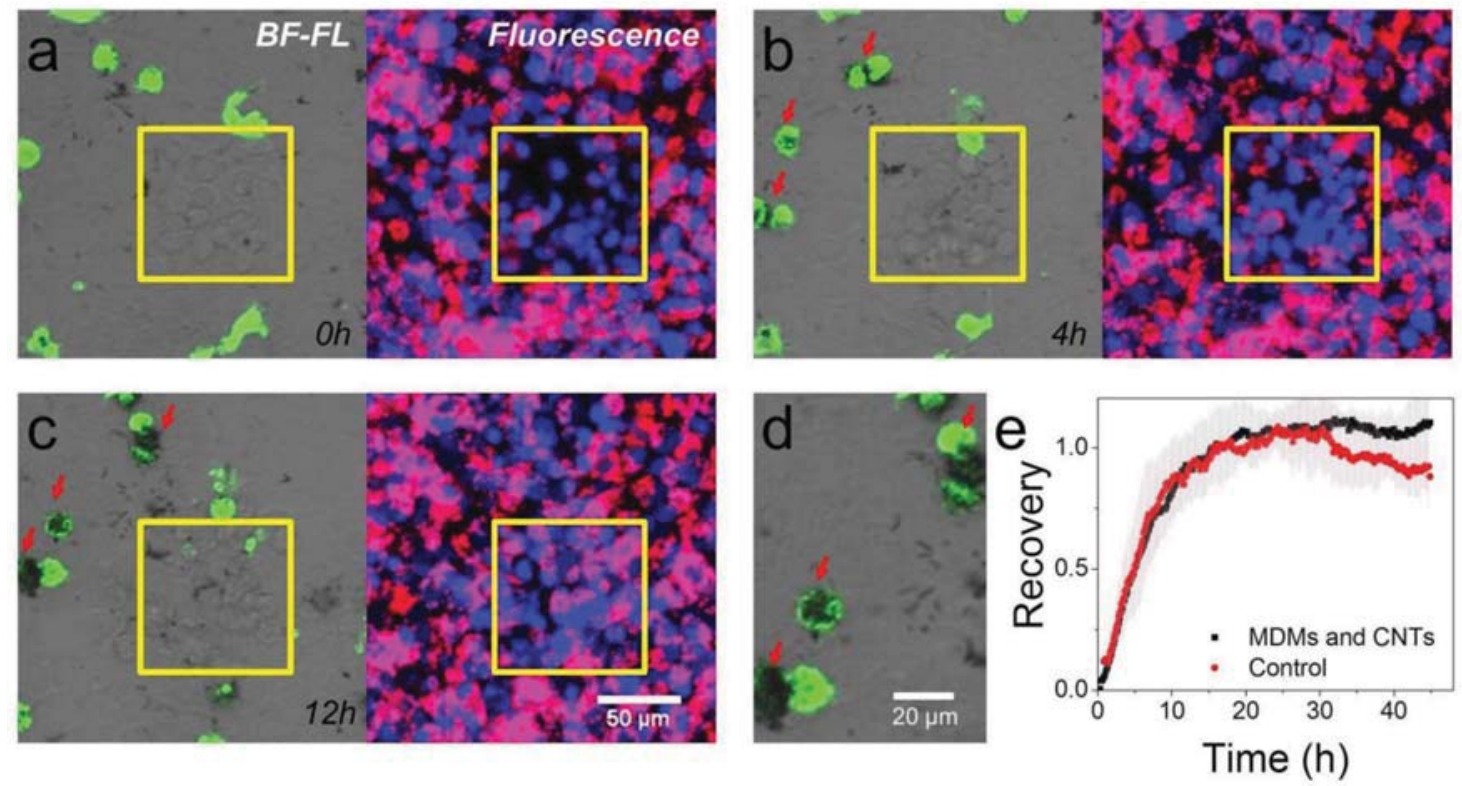

Figure 5. a-c) Selected frames of time-lapse bright-field/fluorescence (BF-FL, left) and fluorescence (right) images at: a) 0 , b) 4 , and c) 12 h after injury showing the clearance of CNTs (black) by macrophages (green); compartmentalization of CNTs inside macrophages was observed (red arrows). Invasion of epithelial cells to the injury sites was visualized but the activity of MDMs in clearance the apoptotic cells was rarely observed. In red and blue are the cell cytoplasm and nuclei, respectively. d) Enlargement view of panel (c). e) Comparison of epithelial injury recovery kinetics in the presence of MDMs and CNTs (black) and the control (only epithelial cells, red). 
and $12 \mathrm{~h}$ after injury) of the internalization of CNTs by the coculture (i.e., MDMs in green on the epithelial cell carpet in red and blue). CNTs appear dark due to their strong light absorption (see also Videos S10 and S11, Supporting Information). Our finding shows that CNTs are mainly internalized by macrophages (i.e., round shape cells) rather than by the epithelial cells (Figure 5c,d). Quantitative analysis shows that in the presence of CNTs and MDMs, the wound healing response was completely restored; a similar rate of recovery to the control experiments was obtained (Figure 5e). We attributed this phenomenon to two concurrent processes: restoration of epithelial cell migration due to the low amount of uptake of CNTs by epithelial cells (since majority of CNTs were internalized by macrophages) and competition between individual epithelial cells and macrophages inside the injury area was hindered as macrophages are only actively clearing the CNTs in the proximity. This can be understood through the higher elevation of IL8 production by macrophages during CNT uptake (Figure S14, Supporting Information).

In summary, we designed a novel system able to closely mimic the epithelial injury and wound healing response. Currently, the majority of assays use mechanical removal of cells, whereas our approach allows damaging a spatially defined region by extensive photobleaching and thus leaves the (apoptotic) dead cells in place. We have shown experimentally and theoretically the effect and contribution of CNTs and macrophages in pulmonary epithelial wound healing. Both the retardation and restoration of wound recovery in the presence of CNTs and MDMs were successfully demonstrated. This result brings us closer to understanding the early development of pulmonary fibrosis induced by CNTs in a wound healing context.

The number of in vitro and in vivo toxicity studies of nanomaterials has increased dramatically over recent past decades. Unfortunately, the availability of information regarding alteration of cell mechanics due to interaction with nanomaterials is still limited. Our current result has shown that nanomaterials such as CNTs can indeed modulate the mechanical behavior of the cells, in a wound healing paradigm. We argue that cell mechanics-based assays can provide important information regarding toxicity of nanomaterials. By combining our approach, with classical viability studies, we hope to be able to provide powerful tools to understand and thereby asses the hazard and safety of nanomaterials.

\section{Experimental Section}

All experiments were performed in triplicate, unless otherwise notified.

Cell Culture: Human alveolar type II epithelial cells (A549), were purchased from LGC Standards (UK) and cultured in complete cell culture media containing RPMI 1640 (Gibco, Life Technologies Europe B.V., Zug, Switzerland) supplemented with $10 \%$ (v/v) fetal bovine serum (PAA Laboratories, Chemie Brunschwig AG, Basel, Switzerland), 1\% (v/v) L-glutamine (Life Technologies Europe), and 1\% (v/v) penicillin/ streptomycin (Gibco) at $37{ }^{\circ} \mathrm{C}$ with $5 \%$ of $\mathrm{CO}_{2}$ for $48-72 \mathrm{~h}$ until reaching $80-90 \%$ cell confluency. Human blood MDMs were isolated from buffy coats provided by the blood donation service SRK Bern and purified using CD14 Microbeads (Milteny Biotech) following a procedure reported previously. ${ }^{[31]}$ Macrophages were grown in the media complemented with the growth factors (M-CSF, $10 \mathrm{ng} \mathrm{mL}^{-1}$ ) allowing cells to differentiate on a six-well plate for at least one week before being used.

Imaging of Epithelial Injury Recovery: Approximately 250000 epithelial cells were seeded 3 days in advance in a four-well Thermo Scientific Nunc Lab-Tek II Chambered Coverglass (Nunc, Denmark; surface area $1.7 \mathrm{~cm}^{2}$; final cell number 1 million) and fluorescently labeled in the next day with Hoechst 33342 and Vybrant DiD (Invitrogen, Germany) following the protocols provided by manufacturers for $50 \mathrm{~min}$. Cells were washed with PBS $1 \times$ for five times and $1 \mathrm{~mL}$ of fresh culture media were added. The induced injury/wound was generated using an extensive photobleaching module available on the Zeiss LSM confocal laser scanning inverted microscope set up at 20× magnification, numerical aperture 0.8 of Zeiss LCI Plan-NEOFLUAR objective lens (Zeiss $\mathrm{GmbH}$, Germany) with a built-in chamber for live cell imaging (i.e., $37^{\circ} \mathrm{C}$ and $5 \%$ of $\mathrm{CO}_{2}$ ). Shortly, small injury sites with rectangular shape corresponds to five different sizes (e.g., $50 \times 50$ pixels, $75 \times 75$ pixels, $100 \times 100$ pixels, $150 \times 150$ pixels, and $200 \times 200$ pixels, with 1 pixel $=0.83 \mu \mathrm{m}$ ) were produced by illuminating continuously the selected area with $405 \mathrm{~nm}$ laser (power $\approx 30 \mathrm{~mW}$ ) for $\approx 23 \mathrm{~min}$ (i.e., acquisition time $1.94 \mathrm{~s}$ and 1000 bleaching repetitions). To visualize apoptotic cells after UV laser illumination, staining with DAPI (4',6-diamidino-2-phenylindole, dihydrochloride), a membrane impermeable fluorophore, or Annexin $V$ was performed. Time-lapse and z-stack images were performed by acquiring images for $10 \mathrm{~min}$ each with $2 \mu \mathrm{m}$ of stack thickness over a duration of $48-72 \mathrm{~h}$. Images were recorded with a frame size of $512 \times 512$ pixels and processed by using maximum intensity projection images using Zeiss Zen 2011 software (Zeiss GmbH, Germany).

Quantification of Recovery Rate: Image processing to quantify the recovery rate was performed using a self-written Matlab code. Briefly, the invasion of live cells into the injury site over time was determined by measuring the fluorescent signal (i.e., pixel area) of the Vybrant DiDlabeled cells. The image processing algorithm included three main steps: 1) intensity thresholding, 2) binarization, and 3) surface area calculation. The value of the pixel area over time was then subtracted from the background value which is obtained at $t=0 \mathrm{~h}$. Please note that this background value comes from partially bleached Vybrant DiD from the dead cells. Normalization of the recovery rate was performed by comparing the obtained value with the average control value attained from a randomly chosen nonbleached area. Data are shown as mean values.

Imaging of Epithelial Injury Recovery in the Presence of Macrophages: Approximately 1 million epithelial cells on a four-well coverglass were stained with Hoechst 33342 and Vybrant DiD as previously described. Meanwhile, MDMs were harvested by gently scraping the cells from the well plates which were subsequently collected in a $15 \mathrm{~mL}$ centrifuge tube. The cells were then centrifuged for 3-5 $\mathrm{min}$ and the supernatant was removed. Fresh media containing Vybrant Dil dye (Invitrogen, Germany) was added to the cell pellet and the cells were stained in the suspension for $\approx 25 \mathrm{~min}$. The cells were washed three times with fresh culture media by repeatedly pelleting the cells and removing the supernatant by means of centrifugation before resuspension in fresh media. Approximately 150000 cells were gently added to the A549 epithelial carpet and the cells were left to sediment for $30 \mathrm{~min}$ before the imaging experiment. Injury was generated as previously described and the corresponding site was carefully chosen to be as close as possible to where MDMs reside but without killing the MDMs itself (i.e., at a distance more than $50 \mu \mathrm{m}$ from/to the wound). Cell (MDMs) tracking was performed using CellTracker software developed by Horvath and Piccinini (http://www. celltracker.website/index.html). ${ }^{[32]}$ The recovery rate was determined as previously described.

Modeling of Epithelial Injury Recovery: See the Supporting Information for details.

CNT Sample Preparation and Characterization: Mitsui-7 multiwalled carbon nanotubes (Mitsui \& Co., Ltd.) were kindly provided by Prof. Vicki Stone, Heriot-Watt University, Edinburgh, UK and used without any further purification. $50 \mu \mathrm{g} \mathrm{mL} \mathrm{m}^{-1}$ of CNT stock solution was stabilized in $1 \mathrm{mg} \mathrm{mL}^{-1}$ BSA (Sigma-Aldrich, Germany) in milliQ water and the solution was sonicated for $3 \mathrm{~h}$. The stock solution was redispersed in 
culture media at 5 and $10 \mu \mathrm{g} \mathrm{mL}^{-1}$. CNTs were analyzed by transmission electron microscopy. Briefly, CNT suspensions were spin-coated and dried on carbon-film square-mesh copper grids (Electron Microscopy Sciences, CF-300-Cu). Electron images were taken with a Tecnai Spirit transmission electron microscope (FEl, Frankfurt, Germany), operating at $120 \mathrm{kV}$. Images were recorded at a resolution of $2048 \times 2048$ pixels (Veleta CCD camera, Olympus, Volketswil, Switzerland).

Imaging of Epithelial Injury Recovery in the Presence of Carbon Nanotubes: Approximately 1 million epithelial cells in a four-well glass bottom dish were stained with Hoechst 33342 and Vybrant DiD as previously described. Cells were cultured in media containing 5 or $10 \mu \mathrm{g} \mathrm{mL}$ CNTs and injury-imaging experiment was conducted subsequently.

In Vitro Experiment to Visualize the Cellular Association of CNTs: Approximately 20000 cells were seeded in each well (surface area $0.7 \mathrm{~cm}^{2}$ ) of a Falcon 8 well chamber slide (Corning Inc., USA) and the cells were grown overnight. Next, $0.5 \mathrm{~mL}$ of 5 or $10 \mu \mathrm{g} \mathrm{mL}^{-1}$ of CNTs in culture media was added and the cells were incubated for another $24 \mathrm{~h}$. The cells were washed five times with PBS to remove noninteracting CNTs. The cell monolayer was fixed with $4 \%$ paraformaldehyde (PFA) in PBS $1 \times$ and followed by immunofluorescence staining. The cells were washed twice with PBS and rinsed in 0.1\% Triton X-100 (Sigma-Aldrich) in PBS for 5 min and subsequently in 1\% BSA (Sigma-Aldrich) in PBS for another $20 \mathrm{~min}$. The cell layer in glass chambers were stained with rhodamine phalloidin (Invitrogen) and DAPI (for visualization of the cellular F-actin cytoskeletons and cell nuclei) for $20 \mathrm{~min}$ in the dark at room temperature followed by washing with PBS. The cover slips were mounted onto glass slides for microscopy measurements. The samples were visualized using a 100x objective lens, numerical aperture 0.8 for enhanced darkfield microscopy and 1.3 for fluorescence in Cytoviva dual mode fluorescenceenhanced darkfield microscopy setup (Cytoviva Inc., Auburn, USA).

In Vitro Dosimetry of CNTs: To quantify the amount of cellular associated CNTs, dosimetry experiment via microwave treatment combined with UV-vis-NIR spectroscopy ${ }^{[23]}$ was conducted. Briefly, in order to keep the number of the cells at each time point (e.g., 4, 24, and $48 \mathrm{~h}$ ) similar, a different number of cells was seeded in the experiment. Between 100000 and 400000 A549 cells were cultured overnight on a 12-well plate (surface area $3.8 \mathrm{~cm}^{2}$ ). Next, $1.5 \mathrm{~mL}$ of 5 or $10 \mu \mathrm{gL}^{-1}$ of BSA stabilized CNTs in CCM was added and the cells were incubated for 4,24 , and $48 \mathrm{~h}$. The cells were washed three times with PBS to remove access of noninteracting CNTs and then fixed with 4\% PFA in PBS. The washing process was repeated twice and $1 \mathrm{~mL}$ of fresh PBS was added gently on the cells. The cells-CNTs mixture were then subjected to the microwave treatment and measured by UV-vis-NIR spectroscopy as described in the literature. ${ }^{[23]}$

IL8 Chemokine Secretion: Approximately 1 million epithelial cells were cultured in a four-well coverglass containing $1 \mathrm{~mL}$ of culture media and subjected to addition of either MDMs, $10 \mu \mathrm{g} \mathrm{mL}^{-1}$ of CNTs or both combination. Samples were grouped into four different types: (1) epithelial cells only (E), (2) epithelial and MDMs (E + M), (3) epithelial cultured in CNTs containing media, and (4) epithelial and MDMs cultured in CNTs containing media, and injury experiments as previously described were performed on each samples. It is important to note that in this particular experiment, the wound size was increased to $691.89 \mu \mathrm{m} \times 691.89 \mu \mathrm{m}$ (with a total surface area of $0.48 \mathrm{~cm}^{2}$ ). The samples were then maintained inside the cell incubator at $37{ }^{\circ} \mathrm{C}$ and $5 \% \mathrm{CO}_{2} .250 \mu \mathrm{L}$ of each media were collected directly after wound $(0 \mathrm{~h})$ with another $250 \mu \mathrm{L}$ removed after $24 \mathrm{~h}$. IL8 secretion was assessed using the commercially available DuoSet ELISA Development diagnostic kit (R\&D Systems, Switzerland), according to manufacturer's protocol. Similar sets of cells without injury treatment (i.e., cultured in the incubator) served as the positive control. Data are shown in a box chart. A parametric one-way analysis of variance (ANOVA) was performed. Values were considered significant $(*)$ in comparison to each control cells if $p<0.05$

In Vitro Visualization of Adhesion and Cytoskeleton Dynamics in the Presence of CNTs: Approximately 20000 epithelial cells were seeded and grown overnight on a Falcon8 well chamber slides. Next, $0.5 \mathrm{~mL}$ of 5 or $10 \mu \mathrm{g} \mathrm{mL}^{-1}$ of CNTs in culture media was added and the cells were incubated for another 4 and $24 \mathrm{~h}$. The cells were washed five times with PBS to remove access of noninteracting CNTs. The cell monolayer was fixed with 4\% PFA in PBS $1 \times$ and followed by immunofluorescence staining. The cells were washed twice with PBS and rinsed in $0.1 \%$ Triton $\mathrm{X}-100$ in PBS for $5 \mathrm{~min}$ and subsequently in 1\% BSA in PBS for another 20 min. Rabbit monoclonal antipaxillin antibody (abcam, Germany) was incubated following the protocols provided by the company for $1 \mathrm{~h}$ and followed by addition of goat antirabbit DY488 secondary antibody and rhodamine phalloidin and DAPI for 25 min for visualization of paxillin focal adhesions, F-actin cytoskeletons and cell nuclei. Cells were washed three times and the cover slips were mounted onto glass slides for microscopy measurements. Samples were scanned using the Zeiss LSM confocal laser scanning inverted microscope set up with $63 \times$ magnification.

Quantification of Paxillin Area: The size of paxillin focal adhesions was quantified by image processing using ImageJ (NIH, USA). The image processing algorithm followed three main steps: (1) intensity thresholding, (2) binarization, and (3) surface area calculation. Data from two conditions: cell cultured with and without CNTs (i.e., control) is shown as average area of paxillin and its $95 \%$ confidence interval. A parametric one-way analysis of variance (ANOVA) was performed. Values were considered significant $(*)$ in comparison to untreated cells (i.e., control) if $p<0.05$.

Quartz Crystal Microbalance to Study Adhesion Dynamics: The adhesion dynamics of A549 epithelial monolayers was monitored in real time using an open-source QCM system (OpenQCM, Rome, Italy). Briefly, a final number of 250000 cells were cultured on optically polished, gold-coated quartz crystal substrates $(10 \mathrm{MHz})$ at $37{ }^{\circ} \mathrm{C}$ and $5 \% \mathrm{CO}_{2}$. The sample was then placed inside a QCM and fresh media was injected through a fluidic system at a flow rate of $166 \mu \mathrm{L} \mathrm{min}^{-1}$ until media covered the entire surface of QCM-cells. Oscillation frequency of the QCM was recorded overnight and next, media containing CNTs $\left(10 \mu \mathrm{g} \mathrm{mL}^{-1}\right)$ was circulated at the same flow rate. The injection was stopped once the cells were covered by the mixture and the QCM response on cell was monitored for the next $48 \mathrm{~h}$. The data are presented as average frequency change $(\Delta f)$ (i.e., the difference of frequency before and after injection of CNTs) and standard deviation.

Cellular Traction Force Microscopy by Micropillar Assays: To measure traction force exerted by cells, micropillar assays ${ }^{[30]}$ developed by Microduits $\mathrm{GmbH}$, Switzerland were used. Briefly, 500000 A549 cells were cultured in a six-well plate overnight (three wells in total). Next, cells were incubated with CNTs with two different concentrations: 5 and $10 \mu \mathrm{g} \mathrm{mL}^{-1}$ and one well was cultured without CNTs (i.e., control cells) for $24 \mathrm{~h}$. After incubation, the cell layers were washed ten times with PBS $1 \times$ to ensure that unbound CNTs were removed. $200 \mu \mathrm{L}$ of Trypsin was added for $5 \mathrm{~min}$ to detach the cell from the well substrate and detached cells were suspended in fresh culture media. Preparation and quantification of traction force was done by following described protocols reported in the literature. ${ }^{[30]}$ In total, around 20000 cells in $1 \mathrm{~mL}$ culture media were gently added above the micropillars and grown overnight. Cells were fixed using 4\% PFA and stained with Brilliant Cresyl Blue ALD dye (Sigma-Aldrich, Germany) for $90 \mathrm{~s}$ followed by five washes with water. The sample was kept in water and imaging was performed using a Leica DMI6000B bright-field optical microscope (Leica, Germany) with a dry 40x magnification objective (numerical aperture 0.6 ). Images were recorded as RGB images with the frame size $1392 \times 1040$ pixels. Displacement of micropillars due to the force exerted by the cells were determined using Mechprofiler software developed by Microduits $\mathrm{GmbH}$ and calculation of the average force was based on the following literature. ${ }^{[30]}$ All physical parameters (e.g., length, diameter and spring constant of the arrays) needed for the quantification were provided by Microduits $\mathrm{GmbH}$. Data were shown as average force per pillar. A parametric one-way analysis of variance (ANOVA) was performed. Values were considered significant $(*)$ in comparison to untreated cells if $p<0.05$. The measured samples were further left dried in room temperature for 1 day and sputtered with gold for scanning electron microscopy visualization. Electron images were acquired using Tescan Mira3 LM FE (Tescan, USA). 


\section{Supporting Information}

Supporting Information is available from the Wiley Online Library or from the author.

\section{Acknowledgements}

The authors kindly thank Hana Barosova, Dr. Roman Lehner, and Laetitia Haeni for blood isolation, CNT sample preparation, and ELISA measurements. The authors would like to acknowledge the Swiss National Science Foundation (310030_159847/1), Peta International Science Consortium, and the Adolphe Merkle Foundation for financial support for the project. The work including primary immune cells white blood cells (monocytes) isolated from human blood was approved by the committee of the Federal Office for Public Health Switzerland (reference number: 611-1, Meldung A110635/2) for AMI.

\section{Conflict of Interest}

The authors declare no conflict of interest.

\section{Keywords}

carbon nanotubes, epithelial injury recovery, epithelial mechanics, macrophage clearance, pulmonary fibrosis

[1] F. Kheradmand, H. G. Folkesson, L. Shum, R. Derynk, R. Pytela, M. A. Matthay, Am. J. Physiol. 1994, 267, L728.

[2] J. R. Lange, B. Fabry, Exp. Cell Res. 2013, 319, 2418.

[3] A. Brugues, E. Anon, V. Conte, J. H. Veldhuis, M. Gupta, J. Colombelli, J. J. Munoz, G. W. Brodland, B. Ladoux, X. Trepat, Nat. Phys. 2014, 10, 683.

[4] A. Datta, C. J. Scotton, R. C. Chambers, Br. J. Pharmacol. 2011, 163, 141.

[5] C. C. Liang, A. Y. Park, J. L. Guan, Nat. Protoc. 2007, 2, 329.

[6] D. P. Kiehart, C. G. Galbraith, K. A. Edwards, W. L. Rickoll, R. A. Montague, J. Cell Biol. 2000, 149, 471.

[7] M. Felder, P. Sallin, L. Barbe, B. Haenni, A. Gazdhar, T. Geiser, O. Guenat, Lab Chip 2012, 12, 640.
[8] M. Pacurari, I. May, P. B. Tchounwou, Environ. Toxicol. 2017, 32, 445.

[9] A. Roycroft, R. Mayor, Cell. Mol. Life Sci. 2016, 73, 1119.

[10] W.-X. Kuai, Q. Wang, X.-Z. Yang, Y. Zhao, R. Yu, X.-J. Tang, World J. Gastroenterol. 2012, 18, 979.

[11] D. Drasdo, R. Kree, J. S. McCaskill, Phys. Rev. E 1995, 52, 6635.

[12] E. Fong, S. Tzlil, D. A. Tirrell, Proc. Natl. Acad. Sci. USA 2010, 107, 19302.

[13] K. M. Adlerz, H. Aranda-Espinoza, H. N. Hayenga, Eur. Biophys. J. 2016, 45, 301

[14] C. Grabher, A. Cliffe, K. Miura, J. Hayflick, R. Pepperkok, P. Rorth, J. Wittbrodt, J. Leukocyte Biol. 2007, 81, 263.

[15] K. B. Baumgartner, J. M. Samet, C. A. Stidley, T. V. Colby, J. A. Waldron, Am. J. Respir. Crit. Care Med. 1997, 155, 242.

[16] R. Hubbard, S. Lewis, K. Richards, I. Johnston, J. Britton, Lancet 1996, 347, 284.

[17] J. D. Byrne, J. A. Baugh, Mcgill. J. Med. 2008, 11, 43.

[18] R. R. Mercer, A. F. Hubbs, J. F. Scabilloni, L. Wang, L. A. Battelli, S. Friend, V. Castranova, D. W. Porter, Part. Fibre Toxicol. 2011, $8,21$.

[19] H. B. Schiller, I. E. Fernandez, G. Burgstaller, C. Schaab, R. A. Scheltema, T. Schwarzmayr, T. M. Strom, O. Eickelberg, M. Mann, Mol. Syst. Biol. 2015, 11, 819.

[20] S. Chortarea, M. J. D. Clift, D. Vanhecke, C. Endes, P. Wick, A. Petri-Fink, B. Rothen-Rutishauser, Nanotoxicology 2015, 9, 983.

[21] L. García-Hevia, R. Valiente, J. L. Fernández-Luna, E. Flahaut, L. Rodríguez-Fernández, J. C. Villegas, J. González, M. L. Fanarraga, Adv. Healthcare Mater. 2015, 4, 1640.

[22] R. Ananthakrishnan, A. Ehrlicher, Int. J. Biol. Sci. 2007, 3, 303.

[23] D. Septiadi, L. Rodriguez-Lorenzo, S. Balog, M. Spuch, H. Barosova, S. Chortarea, M. J. D. Clift, J. Teeguarden, M. Sharma, A. Petri-Fink, B. Rothen-Rutishauser, unpublished.

[24] D. Septiadi, F. Crippa, T. L. Moore, B. Rothen-Rutishauser, A. Petri-Fink, Adv. Mater. 2018, 30, 1704463.

[25] X. Wu, Y. Tan, H. Mao, M. Zhang, Int. J. Nanomed. 2010, 5, 385.

[26] R. Pati, I. Das, R. K. Mehta, R. Sahu, A. Sonawane, Toxicol. Sci. 2016, 150, 454.

[27] D. M. Gryte, M. D. Ward, W. S. Hu, Biotechnol. Prog. 1993, 9, 105.

[28] G. Wang, A. H. Dewilde, J. Zhang, A. Pal, M. Vashist, D. Bello, K. A. Marx, S. J. Braunhut, J. M. Therrien, Part. Fibre Toxicol. 2011, $8,4$.

[29] I. Schoen, W. Hu, E. Klotzsch, V. Vogel, Nano Lett. 2010, 10, 1823.

[30] N. Goedecke, M. Bollhalder, R. Bernet, U. Silvan, J. Snedeker, J. Vis. Exp. 2015, 105, 53350.

[31] S. Steiner, J. Czerwinski, P. Comte, O. Popovicheva, E. Kireeva, L. Müller, N. Heeb, A. Mayer, A. Fink, B. Rothen-Rutishauser, Atmos. Environ. 2013, 81, 380.

[32] F. Piccinini, A. Kiss, P. Horvath, Bioinformatics 2016, 32, 955. 